\title{
Number of Prior Chemotherapies
}

National Cancer Institute

\section{Source}

National Cancer Institute. Number of Prior Chemotherapies. NCI Thesaurus. Code C16159.

Number of prior chemotherapy reg imens: If a patient has received a chemotherapy regimen, provide the number of different single or multi agent chemotherapy regimens the patient has received. A regimen is described as a distinctive collection of agent(s) and/or modalities to be utilized together during a cycle or course of therapy. A chemotherapy regimen may have been discontinued for any reason (e.g. completion of therapy, toxicity, disease progression). If the planned treatment is ABVD/MOPP code as one chemotherapy regimen. 